Revue des patrimoines

$42 \mid 2020$

Imagerie numérique et patrimoine culturel :

représentation et transmission des connaissances

\title{
L'éphémère et les secondes vies des objets du carnaval
}

L'exemple niçois

Short life spans and second lives of carnival works. The example of the Nice

Carnival

\section{Antonin Chabert}

\section{(2) OpenEdition}

\section{Journals}

Édition électronique

URL : http://journals.openedition.org/insitu/27917

DOI : 10.4000/insitu. 27917

ISSN : 1630-7305

Éditeur

Ministère de la Culture

Référence électronique

Antonin Chabert, «L'éphémère et les secondes vies des objets du carnaval », In Situ [En ligne], 42 | 2020, mis en ligne le 12 juin 2020, consulté le 12 janvier 2021. URL : http://journals.openedition.org/ insitu/27917 ; DOI : https://doi.org/10.4000/insitu.27917

Ce document a été généré automatiquement le 12 janvier 2021.

\section{(c) (i) (9)}

In Situ Revues des patrimoines est mis à disposition selon les termes de la licence Creative Commons Attribution - Pas d'Utilisation Commerciale - Pas de Modification 4.0 International. 


\title{
L'éphémère et les secondes vies des objets du carnaval
}

\author{
L'exemple niçois \\ Short life spans and second lives of carnival works. The example of the Nice \\ Carnival
}

Antonin Chabert

1 Le carnaval de Nice est un événement organisé et financé par la mairie de Nice lors de la saison hivernale. Il se déroule pendant environ deux semaines, au cours des vacances scolaires, et donne lieu à une série d'animations. Héritière d'un modèle festif né à la fin du XIX ${ }^{e}$ siècle, cette festivité peut être définie comme un carnaval-spectacle ${ }^{1}$, dont la vocation touristique est évidente. Dans ce dispositif spectaculaire, la dimension matérielle est centrale: ce carnaval se concrétise avant tout par des défilés (corsi), mettant en scène des objets (chars et groupes de grosses têtes) représentant des personnages. Ils défilent de manière théâtralisée dans l'espace urbain, avec des troupes carnavalesques, devant des spectateurs assis dans des tribunes ou bien debout. Le terme «objets du carnaval» renvoie dans cet article à deux catégories de créations majoritaires: les sculptures, présentes sur les chars, et les grosses têtes. Elles sont construites par des fabricants dénommés "carnavaliers", salariés d'entreprises familiales spécialisées. À Nice, le carnaval est véritablement une commande municipale. Chaque année, le défilé se structure autour d'une thématique choisie par le maire, qui sera déclinée à travers les chars et les grosses têtes. Pour leur réalisation, la mairie procède par appels d'offres, d'abord pour les dessins. Une liste de sujets liés au thème est publiée, des illustrateurs (appelés «imagiers») proposent alors des maquettes. Puis une seconde sélection concerne les carnavaliers, avec la construction des sujets à proprement parler, c'est-à-dire la mise en volume des dessins choisis ${ }^{2}$. Les objets sont ensuite fabriqués avec des matériaux précis : un lot de grosses têtes est conçu avec de la résine polyester à partir d'un moule, les sculptures figurant sur les chars sont en polystyrène renforcées avec des tubes métalliques. Du papier journal ou $\mathrm{du}$ papier kraft sont rajoutés sur le polystyrène pour appliquer la peinture. Pour se diriger, le porteur d'une grosse tête se sert d'une lucarne [fig. 1]. 
2 Le présent article entend renouveler l'approche ethnologique du carnaval par l'analyse du fait matériel, en insistant sur le caractère éphémère des créations, mais en montrant en même temps leur possible seconde vie, dont les usages reflètent finalement le visage d'une fête à part entière ${ }^{3}$.

Figure 1

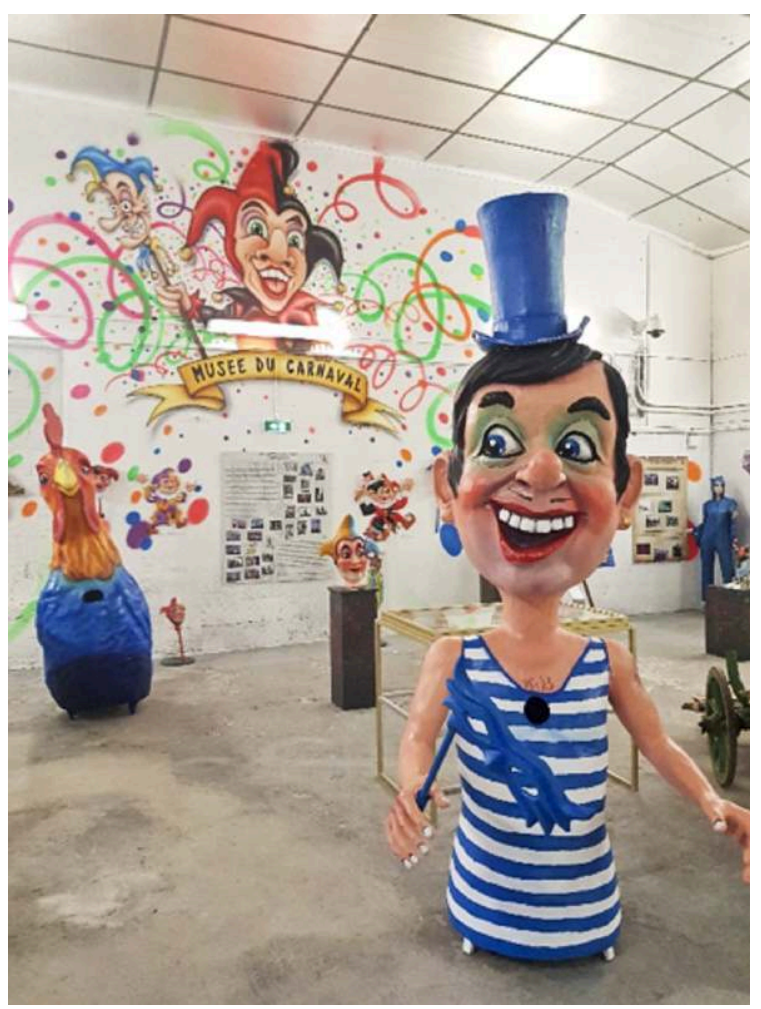

Grosse tête en carton-pâte réalisée par Sébastien Dalbera à partir d'un moule inédit d'Alex Minone. Exposée au musée du carnaval de Nice, à Contes (Alpes-Maritimes).

Photo : Antonin Chabert, 2020.

\section{L'éphémère de l'objet, miroir du cycle de carnaval}

\section{Créer pour ensuite détruire : la mort programmée des objets}

Deux réalités concrètes sont à l'origine de la nécessité de destruction de ces créations : d'une part, le thème du carnaval, modifié tous les ans, qui impose de les recréer, et, d'autre part, leurs dimensions et leurs volumes. Les chars s'élèvent en effet en moyenne à 12 mètres de haut, les grosses têtes mesurent environ 2,50 mètres, sachant qu'elles forment toujours un groupe de huit à douze sujets (un « lot de grosses têtes »). Ces aspects matériels posent bien évidemment des problèmes de stockage quasiment impossibles à surmonter.

L'éphémère de ces objets ne se distingue pas tant par les matériaux utilisés que par la finalité, le sens même de leur existence. Ils sont construits pour un usage et un contexte précis - le carnaval - et sont ensuite stockés dans le hangar des carnavaliers pendant plusieurs mois. À l'exception de certains éléments métalliques et des plateformes des chars, toutes ces productions sont démontées, découpées à la scie sabre 
puis jetées dans des bennes à ordures avant d'être portées à la déchetterie. Les différents matériaux seront brûlés dans un grand incinérateur ${ }^{4}$, terminant leur cycle de vie à l'image du mannequin de carnaval qui se désintègre dans les flammes le soir du Mardi gras. Dans le monde carnavalier, l'éphémère incarne la nature même du carnaval, ce qui amène les fabricants à se désigner comme des "sculpteurs de l'éphémère ». Ils détruisent leurs propres réalisations et sont des créateursdestructeurs.

\section{Acceptation, exaltation, tristesse : le vécu de l'éphémère}

Dans ce contexte, le discours le plus courant est celui de l'acceptation : l'éphémère est inéluctable, intrinsèque au cycle du carnaval. Ce carnavalier âgé de 50 ans, travaillant avec son père, souligne :

Ça fait partie de ma culture de carnaval, depuis tout petit. Les chars, on les a toujours détruits. Le roi, on l'a toujours brûlé.

Ce rapport à l'éphémère s'ancre dans des habitudes, une histoire intime et familiale. La courte vie des objets s'associe à une singularité totalement intériorisée, incorporée, appartenant à l'habitus du constructeur. Éphémère et tradition sont ainsi reliés, l'éphémère symbolise une part essentielle de la tradition carnavalesque à savoir son esprit cyclique. Plus qu'accepté, il est alors revendiqué.

7 Cette nature cyclique fait d'ailleurs référence à la "magie du carnaval », selon les termes des carnavaliers, c'est-à-dire à une réalité fugitive, périssable, une période extraordinaire, qui s'oppose à l'ordinaire du temps routinier. L'éphémère manifeste l'essence du rite carnavalesque, c'est-à-dire un «espace-temps d'identité collective hors de la routine quotidienne individuelle ${ }^{5}$ ». Si cette fugacité incarne, dans ses aspects temporels, le rite en lui-même, le carnavalier endosse le rôle d'acteur, d'officiant du rite. En construisant et en détruisant sa création, il reproduit matériellement le cycle du carnaval. Évoquée de façon exaltée, cette nature éphémère renvoie également à un sens profond, pouvant se lire comme un miroir métaphorique de l'existence humaine :

C'est vraiment un art éphémère. Tu viens au carnaval, tu l'apprécies à fond, tu le regardes, tu te défoules [...]. Ça fait partie fondamentalement de la vie, et y'a toute une notion de vie, de mort dans le carnaval: on se donne un mal fou, à se rendre malade, tout ça pour ça, mais c'est fabuleux ! Ça donne une vraie notion de fragilité à la vie, c'est ça le carnaval ${ }^{6}$.

L'éphémère ramène son créateur à sa propre condition, fragile et passagère. Plus largement, par sa renaissance permanente, l'art du carnaval est décrit comme un art de la vie. La métaphore de la vie revient fréquemment dans les discours relatifs à la naissance d'un nouveau char. Les carnavaliers convoquent souvent l'image de l'accouchement au sujet de la première sortie des chars du hangar, qui attire toujours une foule de passants, de passionnés, d'habitués dans une sorte de premier spectacle. Confectionnés pendant plusieurs mois de gestation, dans la pénombre, les objets sont alors révélés à la lumière du jour en franchissant le seuil de leur matrice. La description de ces instants d'accomplissement, les plus exaltants pour les carnavaliers, témoignent d'un lien fort avec l'objet créé, dans un rapport de "paternité ». Ces indices révèlent que la relation auteur/œuvre est ici réelle, alors qu'il s'agit au départ d'une commande municipale et que les dessins n'ont généralement pas été imaginés par les constructeurs des chars. 
9 Toujours dans le registre de la valorisation, ce mouvement de création / destruction / recréation est dépeint comme l'une des grandes stimulations de l'activité de carnavalier. L'éphémère correspond à une émotion "carburant ", selon la formule de Julien Bernard 7 , à savoir des émotions qui fondent la satisfaction et les motivations d'une profession. Recréer des sujets tous les ans favorise un renouvellement créatif, imaginaire qui procure une émulation et une effervescence, donnant de la saveur et du sens au métier.

Si ces discours d'acceptation et de glorification sont majoritaires, l'obligation de destruction peut toutefois susciter de l'amertume. Certains fabricants mettent en avant leur tristesse, leur nostalgie mêlée d'une certaine impuissance. L'injustice est d'autant plus ressentie que le temps de déconstruction est bref tandis que celui de la conception s'étale pendant plusieurs mois avec un rythme de travail exigeant : "On met des mois à fabriquer les sujets et, en une journée, ils sont démontés! », déplore un carnavalier. La tristesse traduit aussi la fin d'une fête, d'une parenthèse heureuse et le retour à la vie ordinaire, synonyme d'une première nostalgie. Au-delà de la tristesse, un informateur relate le sentiment de révolte qu'il éprouve lors de la démolition des objets, un moment de libération de sa colère :

Tout le temps que tu passes à faire un char et, finalement, tout part à la poubelle.

Tu n'as pas le choix, tu dois tout démonter. Alors, avec agressivité, je casse tout ! Je

tape avec méchanceté.

11 L'intensité des coups donnés aux sculptures illustre la force du ressentiment, en même temps que ces instants ont aussi, plus largement, une fonction d'exutoire. Ce témoignage symbolise en tout cas la révolte face à l'impératif de démolition auquel le carnavalier doit tout de même se soumettre.

Toutes ces émotions négatives (la tristesse, la nostalgie, le ressentiment) démontrent que cet impératif peut être parfois mal vécu. Après tout, détruire sa propre création, n'était-ce pas détruire une partie de soi à travers l'investissement personnel fort qu'exige son temps de fabrication? Celui-ci s'associe à des souvenirs, des tranches de vie partagées avec d'autres, dans un travail d'équipe où les journées sont interminables à l'approche du premier défilé. De plus, offrir ce spectacle à une foule représente une expérience valorisante, où le constructeur est au-devant de la scène, au cœur du spectacle par l'intermédiaire de ses créations. Leur désintégration signifie le retour à l'anonymat et à la banalité de la vie de tous les jours. Cette amertume est sans doute renforcée par le contraste entre l'imaginaire véhiculé par les objets, leur expression féérique ou humoristique, leurs couleurs vives et la manière dont ils sont détruits: jetés dans la froideur et l'anonymat d'une benne à ordure, anéantis dans un incinérateur. C'est l'âme de la fête qui est annihilée soudainement et l'éventail de ses souvenirs, les émotions suscitées auprès des publics, les interactions créées avec les enfants.

13 Face à cette mort programmée, les questions du témoignage et du souvenir sont posées. Un constructeur évoque cette absence de traces et le rôle de la photographie dans ce contexte :

Il n'y a même pas un souvenir, ni rien, tout est détruit. Il n'y a rien pour conserver ou se remémorer. Tous les ans, je photographie pour qu'il me reste des images.

14 Il est fréquent que les carnavaliers s'adonnent à la collection de photographies, moyen unique de pouvoir conserver la mémoire des œuvres, mais aussi de pouvoir s'en 
inspirer. Cette pratique de la photographie comme « reliquaire » se rencontre chez des adeptes d'autres expressions artistiques urbaines éphémères, à l'image du graffiti ${ }^{8}$.

La destinée de la plupart des créations est donc leur disparition après le carnaval. Mais il existe, pour certaines d'entre elles, diverses formes de réemplois, de nouveaux usages qui leur confèrent une nouvelle vie. Cet aspect rappelle le concept de biographie d'objets cher à Arjun Appadurai ${ }^{9}$ ainsi qu'à Igor Kopytoff, qui propose une «biographie culturelle des choses ${ }^{10}$ ». Les objets, à l'instar des personnes, ont des vies sociales : ils subissent des évolutions qui dessinent une trajectoire. Ce regard incite à refuser une vision purement matérialiste et utilitaire des objets pour envisager plusieurs problématiques : les rapports sujets/objets, les goûts esthétiques, les émotions auxquels ils sont reliés, leur valeur intime et mémorielle, leurs changements de perception au cours de leur existence, miroir de leurs changements de statut ${ }^{11}$.

\section{Les réemplois ou les secondes vies des créations}

\section{La revente et le don}

Après la fête, en fonction de la demande, une minorité de chars peut être revendue à des municipalités souhaitant les réutiliser. Plus précisément, ce sont les sculptures des chars qui sont revendues puisque leur plateforme appartient à la mairie de Nice. Ces reventes montrent certes l'aura de ces créations, mais elles signalent aussi l'importance des réseaux, des échanges noués au fil des ans entre les carnavaliers niçois et ceux d'autres villes et plus largement des élus locaux, des responsables de services culturels. Les objets connaissent ainsi une réappropriation par d'autres carnavaliers qui les font revivre, changeant de lieu d'utilisation mais réactivés dans un contexte festif identique (un corso). Un autre type de réemploi assez proche existe à travers le système de la prestation : sollicités par une mairie, les carnavaliers niçois viennent défiler avec leur char. Pour les organisateurs, importer des sujets du carnaval de Nice contribue à créer une fête avec des chars de qualité qui seront appréciés par la population.

17 La seconde vie de ces objets s'illustre également par les dons. Il arrive que les carnavaliers niçois donnent des grosses têtes ou des sculptures de chars à des bénévoles pour des carnavals de villages ou de petites villes. Ces dons permettent de faire perdurer des carnavals mineurs, dans diverses régions de France. Donner est évoqué comme un acte d'échange et de partage procurant de la satisfaction, tel que le souligne ce carnavalier :

Quand on a un petit carnaval qui nous fait une demande, on donne volontiers:

«Vous voulez quoi ? Allez, prenez! » C'est bien que les traditions perdurent, ça fait plaisir. Ça vient du carnaval, ça fait une fête.

18 Le don favorise une future fête de village et donc un instant de convivialité, de joie, qui aide d'autres passionnés ou bénévoles, en même temps qu'il est valorisant pour le donateur. Parfois, le don s'inscrit dans une habitude avec des passionnés qui ont besoin d'objets pour leur propre carnaval. Les familles Povigna ou Durand effectuent ainsi des dons de moules anciens de grosses têtes à un passionné, Sébastien Dalbera, qui a revitalisé le carnaval de son village dans l'arrière-pays niçois. 


\section{Objets itinérants, objets ambassadeurs}

19 Dans certains cas, des chars entiers sont exportés à une échelle géographique très vaste, pour des carnavals ou des festivals de rue. Ces exportations existaient déjà au début $\mathrm{du} \mathrm{xx}^{\mathrm{e}}$ siècle. Elles témoignent de la réputation mondiale qu'a acquis le carnaval de Nice, festivité qui a permis à cette cité de s'imposer comme un "pôle de loisirs sophistiqué $^{12}$ » dès la fin du $\mathrm{xIX}^{\mathrm{e}}$ siècle. Héritière de cette vocation touristique, l'exportation du carnaval remplit aujourd'hui un rôle d'ambassadeur de la destination azuréenne, notamment vis-à-vis des pays asiatiques. En 2017, des chars niçois ont défilé en Chine, à Xiamen, puis en 2018, à Ningbo, une station balnéaire près de Shanghai. Ces deux exemples récents s'inscrivent dans la politique diplomatique et touristique de Nice, jumelée avec ces deux cités chinoises. Depuis plusieurs années, Nice développe une politique d'attractivité envers la clientèle chinoise, en proposant par exemple une programmation événementielle et culturelle ${ }^{13}$. Ce tourisme a été facilité par l'ouverture d'une ligne aérienne directe entre Nice et Pékin, en août 2019. Dans le cadre du carnaval, la clientèle chinoise représente un «segment de clientèle " privilégié en raison de son pouvoir d'achat.

Cette contextualisation n'est pas sans intérêt pour rappeler le sens de la présentation des chars niçois dans le carnaval international de Ningbo, en 2018. Parmi les chars, ceux du couple royal (le roi et la reine de l'édition 2018) ont été choisis par la Ville de Nice pour défiler. Ces chars sont les plus emblématiques, les plus prestigieux et s'imposent chaque année comme les plus grands au sein du corso de Nice $^{14}$. L'esthétique spectaculaire, avec ses chars-emblèmes, est mise au service de la promotion d'une fête et, à travers elle, d'une cité. Si elle signifie un changement de lieu, l'exportation des sujets ne signifie pas forcément une modification radicale de leur contexte d'utilisation: en Chine, les chars ont défilé dans une station balnéaire touristique au sein d'un carnaval-spectacle urbain identique à celui de Nice, comprenant des tribunes. Les productions carnavalesques voyagent ainsi pour promouvoir une cité, devenant les symboles d'une identité, d'une singularité, ce qui souligne leur capital promotionnel. Ce sont des objets ambassadeurs ou « publicitaires».

\section{Les usages décoratifs}

21 La seconde vie des productions carnavalesques s'illustre encore dans des usages décoratifs. Ceux-ci concernent spécifiquement les grosses têtes qui peuvent être réutilisées par des commerçants pour décorer leur magasin. Cette pratique aussi est historique, fréquente au cours du siècle passé. Les grosses têtes sont à présent la propriété de la Ville, qui les stocke après la festivité. Un tri s'opère alors: les exemplaires trop détériorés sont jetés, promis à une disparition intégrale, broyés et désintégrés, tandis que d'autres, peu abîmés, sont conservés. À Nice, divers types de commerces ou d'enseignes les récupèrent et les exposent pendant la période $\mathrm{du}$ carnaval: grandes surfaces, centres commerciaux, cafés, restaurants, ou encore l'aéroport. Pour les commerçants, il s'agit d'agrémenter leur magasin en utilisant une décoration festive, joviale, amusante. Ces grosses têtes se rencontrent généralement dans le centre-ville, devant les boutiques, à proximité de leur entrée, ou positionnées en face. Elles sont de temps en temps disposées par groupe de deux, face à face, ce qui crée une situation de dialogue entre deux personnages, de manière théâtralisée. Ces objets aux couleurs vives, chatoyantes, signalent une boutique et envoient au badaud 
un message de bienvenue. Ce sont des objets « publicitaires » ou « signalétiques ». Dans un hall de grande surface, ils meublent l'espace et rendent plus vivant ce type de lieux réputé froid et sans âme.

Ces usages décoratifs sont encouragés par les organisateurs de la fête à des fins promotionnelles. Pour la Ville de Nice, propriétaire de ces objets, leur diffusion dans la cité représente en effet une occasion de promotion de l'événement dans les rues, les quartiers, les lieux traversés par des flux importants de personnes. Elle confère au carnaval une visibilité, gratuite et efficace, susceptible d'attirer l'attention des enfants - particulièrement "visés " par le marketing de la fête - ou d'éveiller la curiosité des touristes. Dans le hall de l'aéroport de Nice, la grosse tête exposée, avec ses couleurs fantaisistes, accueille l'étranger en lui révélant des valeurs positives associées à la destination : la fête, l'énergie, la créativité, la tradition, en même temps qu'elle apporte de la gaieté dans un espace vide et désincarné, un «non-lieu ${ }^{15}$ ». Les emplacements des grosses têtes ne sont donc pas neutres et correspondent à des lieux commerciaux ou touristiques.

À côté de ces réemplois, d'autres secondes vies sont possibles, motivées cette fois par des dynamiques patrimoniales.

\section{La patrimonialisation de l'éphémère}

Plusieurs sculptures de chars du carnaval de Nice sont aujourd'hui conservées par un musée national, le Musée des civilisations de l'Europe et de la Méditerranée (Mucem), à Marseille. Cette patrimonialisation institutionnelle fait suite à des enquêtes-collectes, dont les objets, imposants, ont été présentés dans une exposition temporaire du musée, "Le monde à l'envers", en 2014. Les formes de patrimonialisation actuellement observées à Nice, au sujet du carnaval, concernent des pratiques patrimoniales informelles, en dehors des structures culturelles officielles. Elles sont portées par des passionnés, "carnavalophiles ${ }^{16}$ ", animées par des motivations émotionnelles et passionnelles. Des auteurs ont déjà analysé ces types de patrimonialisations ordinaires, impulsées par des individus, en marge des institutions étatiques ${ }^{17}$. Pour désigner ce type d'acteurs, je propose la notion d'« entrepreneur en patrimonialisation» - le terme « entrepreneur » ne devant pas être compris ici dans son acception économique : il s'agit d'un initiateur d'une mise en patrimoine, qui s'investit personnellement et financièrement de manière conséquente, en étant guidé par une passion. Ces figures locales, reconnues comme passionnées et expertes, s'impliquent, sensibilisent, tentent de mobiliser, en ayant le sentiment d'accomplir une mission. Elles détiennent des collections de photographies, d'ouvrages, d'objets, acquièrent ou louent des locaux pour les stocker et parfois même créer un musée. Elles «fabriquent» du patrimoine indépendamment des institutions, du moins à l'origine. À Nice, les mobilisations de ces quelques passionnés pour faire ouvrir un musée municipal du carnaval n'ont pour l'heure pas trouvé de financements publics, dans un contexte où ce type de projets n'apparaît pas prioritaire pour le pouvoir local ${ }^{18}$.

Pour illustrer ces initiatives patrimoniales, j'évoquerai l'exemple de Sébastien Dalbera, un « carnavalophile » engagé. Amoureux de la tradition carnavalesque, affectionnant de façon générale le vintage, ce carnavalier trentenaire collectionne depuis plus de dix ans des cartes postales, des photographies anciennes ou encore des mascarons du carnaval de Nice. L'originalité de sa collection provient surtout de ses sculptures 
carnavalesques ainsi que de ses dizaines de grosses têtes. Elles sont stockées dans une grande remise qui s'apparente à une sorte de réserve de musée, dans laquelle la collection s'accroît régulièrement, au fil des dons. Cette accumulation reflète la passion dévoratrice, envahissante, courante dans le monde des collectionneurs. Jacques Hainard et Roland Kaehr l'ont dépeinte à travers les métaphores de l'engrenage et du virus ${ }^{19}$ saisissant le passionné.

Pour exposer ces objets, Sébastien a créé une association et ouvert un musée consacré au carnaval, en juin 2019. Établi à Contes, dans l'arrière-pays niçois, ce musée privé a été financé essentiellement avec ses économies personnelles et de manière secondaire par une souscription lancée sur Internet ${ }^{20}$. Installé dans un ancien entrepôt, avec peu de moyens, le musée garde aussi volontairement son aspect d'origine afin d'immerger le visiteur dans l'ambiance d'un hangar de carnavaliers. L'espace d'exposition présente une partie de la collection du passionné avec notamment des grosses têtes, des sculptures, un char reconstitué. Ces objets sont issus de dons provenant de carnavaliers, d'anciens carnavaliers ou bien de leurs proches qui souhaitent s'en séparer à leur décès faute de places, ou à l'occasion d'un rangement d'une cave, d'un garage... Acteur patrimonial, Sébastien est régulièrement sollicité pour ce type de dons, mais il alimente également sa collection au fil de ses recherches et découvertes. La collection s'enrichit parfois de créations récentes, mais toujours fidèles à une certaine esthétique carnavalesque que le passionné considère comme traditionnelle. Pour décorer l'entrée de son musée, il a d'ailleurs choisi une sculpture carnavalesque de 2019 représentant l'acteur Jean-Paul Belmondo, donnée par des carnavaliers [fig. 2]. Particulièrement voyante, elle signale l'entrée du musée et retient l'attention.

Figure 2

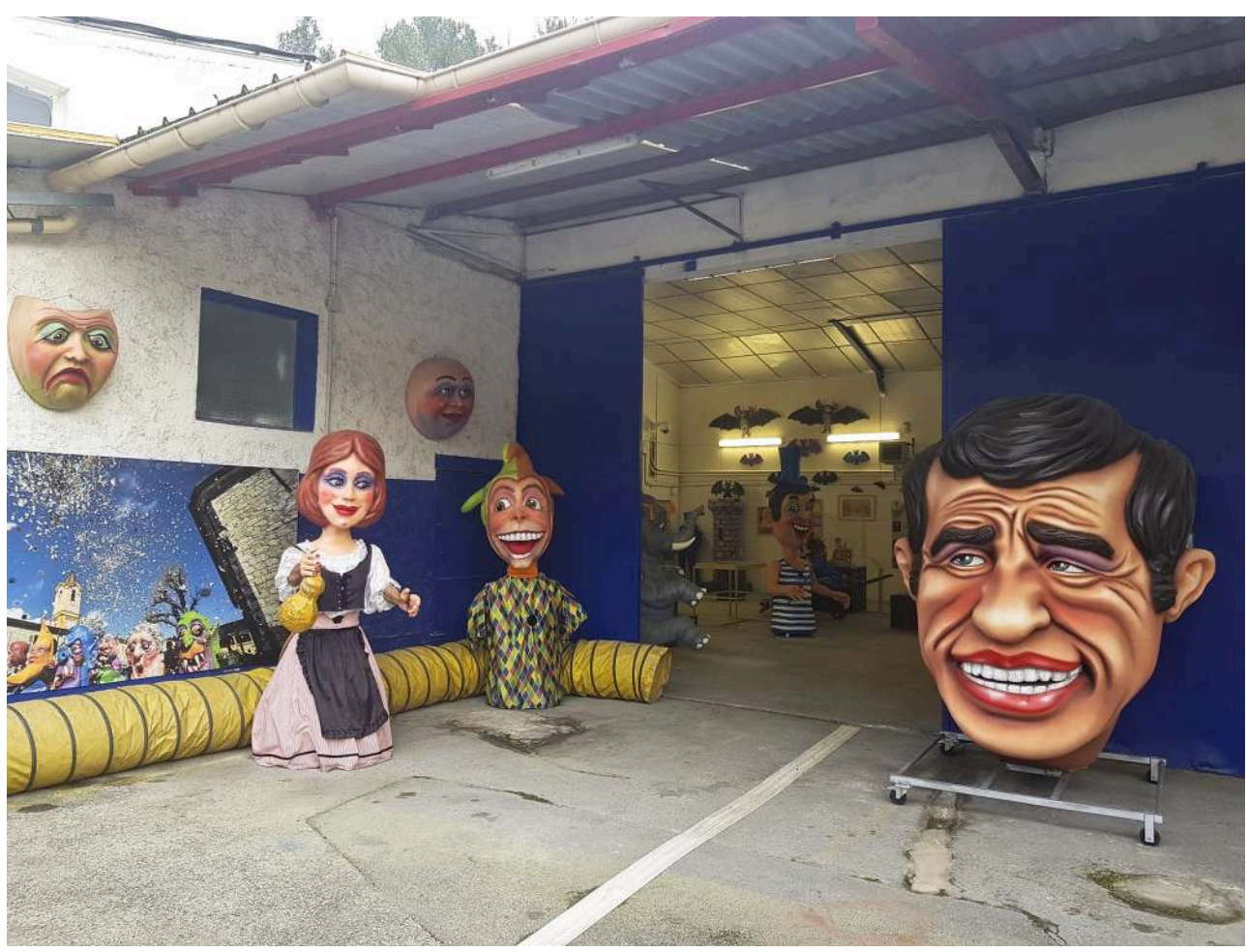

Musée du Carnaval, à Contes (Alpes-Maritimes). Au premier plan, en guise de signalétique, une sculpture représentant Jean-Paul Belmondo, réalisée en 2019 par la société « Nice festivités ». Photo : Antonin Chabert, 2020. 
objets manifestent un style et une esthétique vus comme authentiques: un imaginaire (personnages et motifs), des aspects matériels et esthétiques (formes physiques caricaturées, larges sourires, couleurs vives). Ce sont des " objets-passion ", reliés à une esthétique essentialisée, magnifiée, s'associant à une panoplie d'émotions. Les liens entre émotions et action patrimoniale sont à souligner dans l'édification de ce musée, qui est une reconstruction de l'imaginaire de son collectionneur, du monde de l'enfance et une immersion dans ses souvenirs. En outre, en conservant et en exposant ces objets, Sébastien accomplit également une entreprise de sauvegarde, d'hommage vis-à-vis de leurs auteurs afin de les arracher au néant et à l'oubli définitif. Le musée peut alors être interprété à la manière d'un «temple » à la mémoire des " anciens ». La " mission» du collectionneur est de rendre justice à ces créations encombrantes, déconsidérées, peu valorisées par la société et souvent jetées, en négation de leurs auteurs, de leur vécu, des savoir-faire propres à leur fabrication ${ }^{21}$.

\section{Par-delà la conservation, la réactivation des objets}

Mais la conservation de ces objets est nourrie par une démarche qui va au-delà de la simple volonté de les sauvegarder. Il est en effet fréquent que Sébastien les remette en scène dans le carnaval qu'il organise dans son village de l'arrière-pays niçois, à Châteauneuf-Villevieille. Le passionné a revitalisé cette fête, qu'il revendique comme un «carnaval d'avant, d'il y a trente ans ». Dans une complémentarité, ce militant du patrimoine est aussi un militant de la fête, qui fait donc renaître des objets et les fêtes qui leur sont liées. La renaissance des créations positionne le passionné en tant que passeur et réactivateur. À travers leur nouvelle vie, il fait "revivre » leurs auteurs, souvent tombés dans l'oubli, qu'ils soient pour lui inconnus ou qu'ils incarnent un héritage voire un modèle. Cette tonalité d'hommage est d'autant plus vraie lorsqu'il existe un lien entre l'auteur de l'œuvre et son collectionneur. Par exemple, Sébastien conserve soigneusement la sculpture d'un lion réalisée dans les années 1990 par Alex Minone, aujourd'hui décédé. Il l'a obtenue à la suite d'un don d'un carnavalier qui l'avait lui-même conservée. Alex Minone fut pour Sébastien un mentor qui l'a initié aux arts du carnaval. Dans ce cas, l'objet conservé est un objet d'affection ${ }^{22}$, porteur d'une dimension mémorielle et affective, renvoyant dans le cas précis à un guide, un « maître », pour reprendre un terme du milieu carnavalier d'autrefois. 
Figure 3

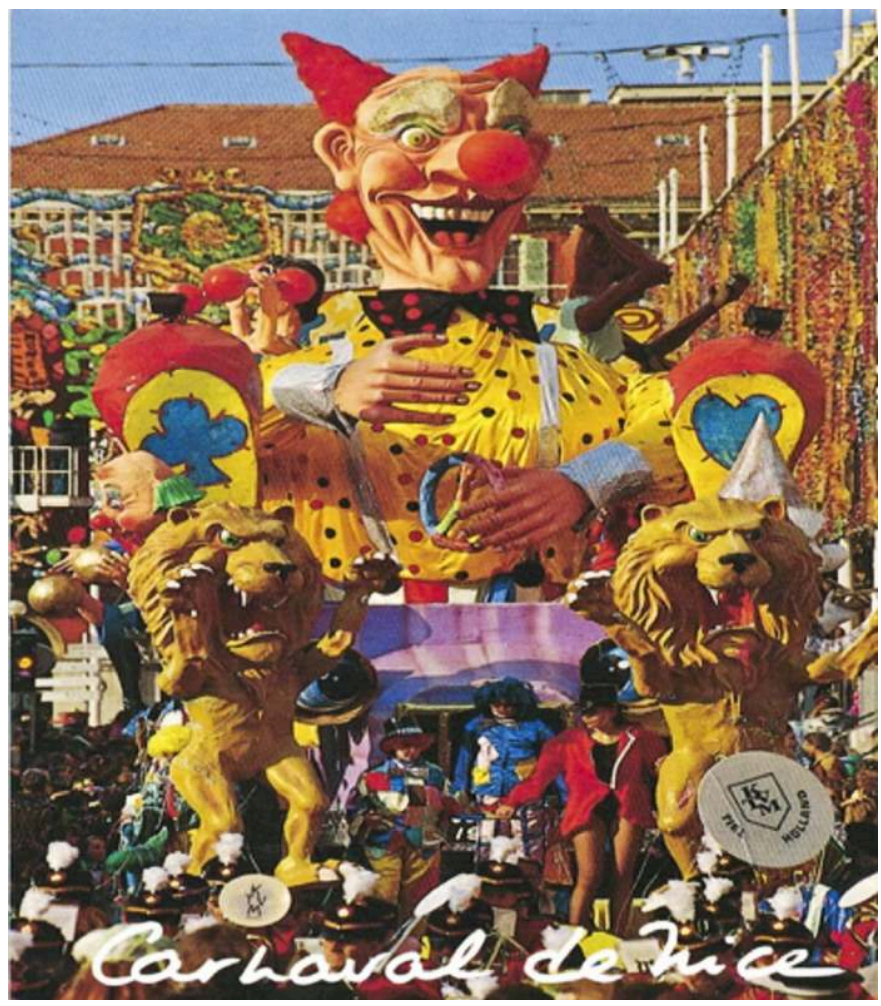

La destinée d'une sculpture du carnaval, construite par Alex Minone : carte postale du carnaval de Nice « Roi des arts », en 1994

(Photographe et éditeur inconnus.)

Figure 4

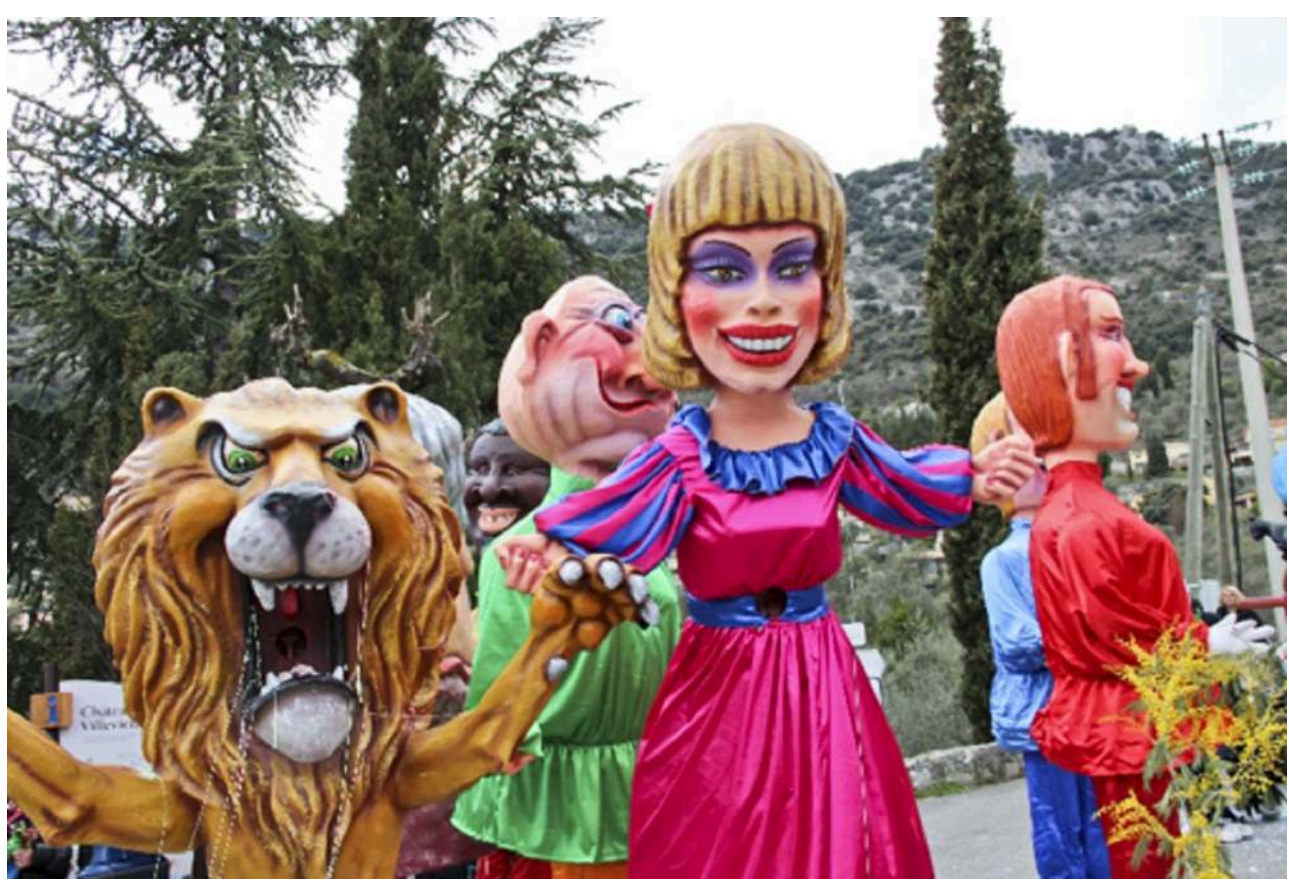

Carnaval de Châteauneuf-Villevieille.

Photo Sébastien Dalbera, 2014. 
Figure 5

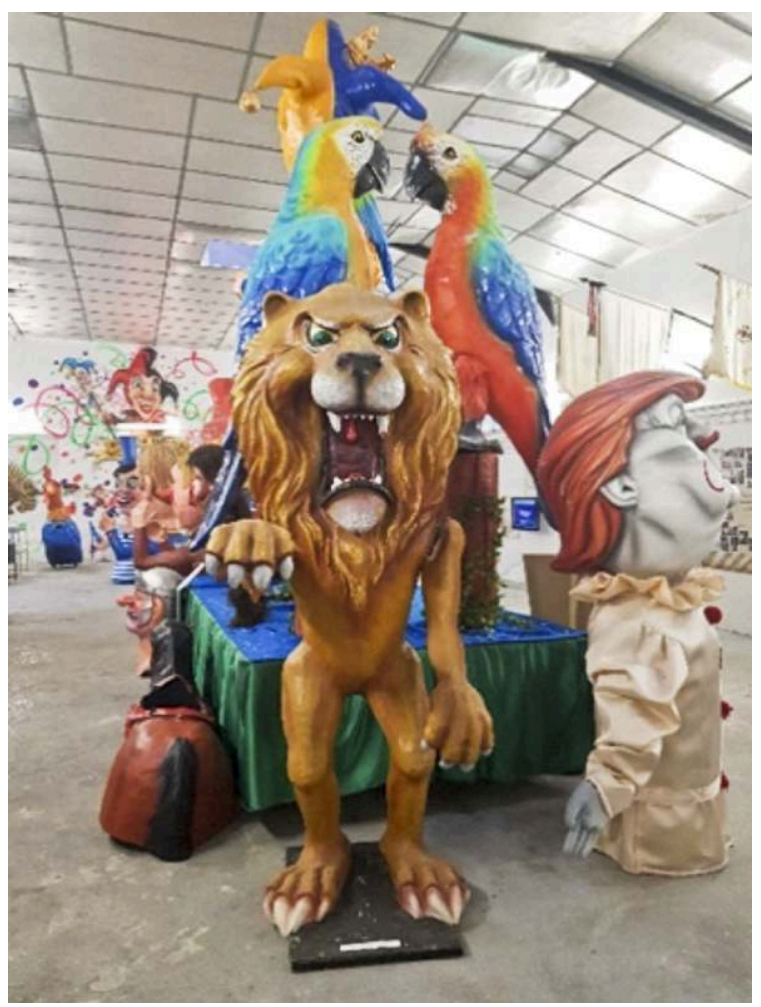

Musée du carnaval de Nice à Contes (Alpes-Maritimes).

Photo : Antonin Chabert, 2020.

La première photographie [fig. 3] montre la première vie de la sculpture du lion, lors de l'édition 1994 du carnaval de Nice $^{23}$. Après l'avoir récupérée, le collectionneur l'a réutilisée plusieurs fois dans le carnaval de son village, avec une nouvelle mise en scène, faisant renaître l'objet et perpétuant l'œuvre de son auteur [fig. 4]. La sculpture est à présent exposée dans son musée [fig. 5], ce qui ne signifie pas la fin de ses usages originels. Elle est en effet mise à l'honneur dans des parades carnavalesques locales, avec d'autres pièces de la collection du musée. Celui-ci peut se définir comme un musée "vivant", où les objets sont susceptibles d'être réemployés, dans des pratiques patrimoniales alternatives, loin des règles académiques en vigueur dans les musées publics bénéficiant de l'appellation " Musée de France». Par ailleurs, la biographie de cette sculpture illustre à nouveau le thème de la circulation de l'objet, qui circule entre des individus, des époques, des usages. Ce type d'objets peut être envisagé comme hybride, évolutif, changeant de "peaux ", de rôles, de catégories et aussi de lieux de présentation a priori antinomiques (salle de musée / parade festive). Sa trajectoire se découpe en plusieurs cycles, dont les derniers sont répétitifs : création, utilisation, sommeil, changements de propriétaires par deux dons successifs, réactivation, mise en exposition, réactivation, remise en exposition...

D'autres productions matérielles conservées font quelquefois l'objet d'une restauration en raison de leur état délabré. Cette grosse tête représentant Picsou donnée par un ancien carnavalier était à l'origine abîmée [fig. 6]. Réalisée en cartes postales, elle a été restaurée à partir de couches de papier journal, de cartes postales, de papier collées grâce à un mélange de farine et d'eau. Elle a été ensuite peinte. 
Figure 6
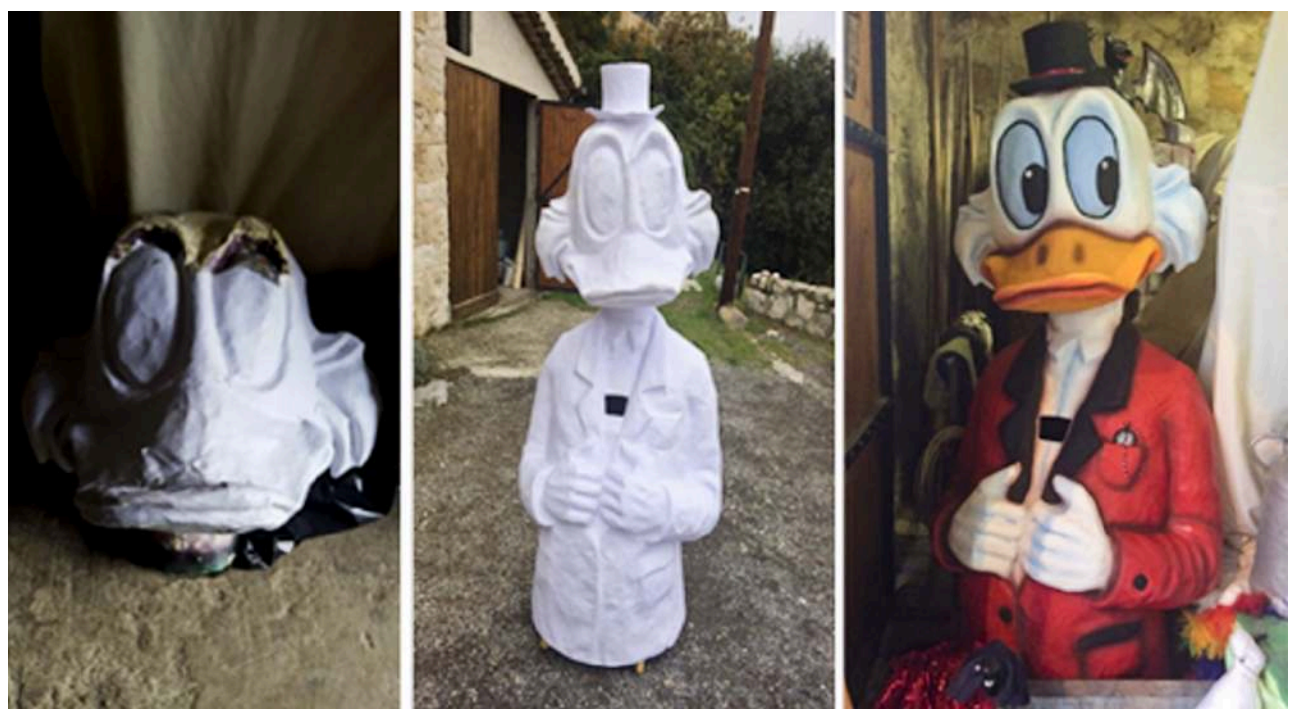

La renaissance de Picsou. Restauration d'une grosse tête par Sébastien Dalbera, carnavalier (Châteauneuf-Villevieille, Alpes-Maritimes).

Photo : Antonin Chabert, 2016.

Le passionné mobilise des techniques qui étaient dominantes au siècle passé, lorsque les carnavaliers n'employaient pas la résine polyester dans la confection des grosses têtes. Il fait revivre des techniques et des matériaux qu'il estime plus nobles que ceux d'aujourd'hui, décriés parce qu'inauthentiques, peu esthétiques, toxiques et polluants. Cette restauration est évoquée comme une relation sensorielle - quasiment sensuelle - avec les matériaux anciens, associée au plaisir de les toucher, de les retravailler, de recourir aux mêmes gestes que les carnavaliers qui ont autrefois fabriqué ces sculptures. Restaurer l'objet relie le passionné à son auteur, ce qui suscite d'autant plus d'émotion qu'il s'agit d'une renaissance. Cette expérience sensorielle et émotionnelle avec le passé renvoie finalement au sens même de la notion de patrimoine définie ainsi par Sylvie Sagnes :

Le patrimoine, c'est le passé que l'on voit, que l'on touche, que l'on écoute, que l'on goûte, que l'on revêt, etc. ${ }^{24}$.

Objets éphémères, "publicitaires ", ambassadeurs, patrimoniaux... À partir des trajectoires de ces objets se dessinent véritablement les grandes dynamiques qui traversent un carnaval urbain contemporain tel que celui de Nice, fait social complexe où s'imbriquent économie, tourisme, patrimoine, esthétiques, émotions et imaginaires.

\section{NOTES}

1. BOYER Marc, L'Hiver dans le midi. L'invention de la Côte-d'Azur. XVIII ${ }^{e}-\mathrm{XXI}{ }^{e}$ siècle, La Tour d'Aigues, Éditions de l'Aube, 2002. 
2. Les dessins (maquettes) peuvent parfois être proposés par des « carnavaliers-imagiers ", mais cela est minoritaire.

3. Cet article s'appuie sur le travail de thèse entrepris par l'auteur: CHABERT Antonin, "Anthropologie du carnaval de Nice. Politiques, professions et esthétiques de la fête », thèse de doctorat d'anthropologie sociale et culturelle soutenue à l'université Aix-Marseille Université, 2019. Le travail d'enquête de terrain, effectué entre 2014 et 2018, a été financé grâce à une allocation du ministère de la Culture (DRPRS, direction des Patrimoines).

4. Certaines pièces métalliques sont conservées, tandis que d'autres sont découpées en petits morceaux puis données à des ferrailleurs. Les autres matériaux sont jetés à la déchetterie puis incinérés. Les carnavaliers sont de plus en plus soucieux du recyclage de leurs déchets; une entreprise de carnavaliers a pour projet d'acquérir une machine pour recycler le polystyrène.

5. AGIER Michel, Anthropologie du Carnaval. La ville, la fête et l'Afrique à Bahia, Paris, Éditions Parenthèses, 2000, coll. « Eupalinos ", série « Culture, histoire et société », p. 230.

6. Citation extraite d'un entretien avec un carnavalier âgé de 38 ans (octobre 2016)

7. BERNARD Julien, La Concurrence des sentiments. Une sociologie des émotions, Paris, Métailié, coll. «Traversées », 2017, p. 164.

8. Sur ce point, voir: THÉVOZ Michel, Art, folie, LSD, graffiti, etc., Lausanne, L'Aire, coll. « Métamorphoses », 1996.

9. APPADURAI Arjun, "Introduction, commodities and the politics of value ", in APPADURAI Arjun (dir.), The Social Life of things. Commodities in cultural perspective, Cambridge, Cambridge University Press, 1986, p. 3-63.

10. KOPYTOFF Igor, "The cultural biographies of things. Commodization as process", in APPADURAI Arjun (dir.), The Social Life of things. Commodities in cultural perspective, Cambridge, Cambridge University Press, p. 64-94.

11. Sur ce point, voir le chapitre «Le projet biographique » dans BONNOT Thierry, L'Attachement aux choses, Paris, CNRS éditions, coll. « Le passé recomposé », 2014, p. 147-153.

12. FERREIRA Felipe, L'Invention du carnaval au XIX siècle. Paris, Nice, Rio de Janeiro, Paris, L'Harmattan coll. « Géographie et cultures », 2014, p. 204.

13. On peut citer le festival de lanternes chinoises «Nice, baie des lumières ", du 29 novembre 2019 au 23 février 2020.

14. En 2014, le char du roi (« roi de la gastronomie ») s'élevait à une taille record de 17 mètres de hauteur.

15. AUGÉ Marc, Non-lieux. Introduction à une anthropologie de la sur-modernité, Paris, Éditions du Seuil, coll. « La librairie du Xx siècle », 1992.

16. Néologisme forgé par l'auteur de cet article pour désigner un passionné du carnaval.

17. Voir par exemple, au sujet de la revitalisation de certaines pratiques musicales au Portugal : ISNART Cyril \& RODRIGUEZ DOS SANTOS José, « Le Mestre et son “cours”. Figure et institution de la transmission du chant dans le sud du Portugal », in ADELL Nicolas \& POURCHER Yves (dir.), Transmettre, quel(s) patrimoine(s) ? Autour du patrimoine culturel immatériel, textes en partie issus des communications de journées d'études (université Toulouse 2 - Le Mirail, 16-18 juin 2010), Paris, M. Houdiard, 2011, p. 167-177.

18. Une Cité internationale du carnaval de Nice a toutefois été annoncée par la Ville de Nice, mais sans qu'un calendrier précis de travaux ne soit pour l'heure dévoilé. Ce projet est encouragé par l'historienne Annie Sidro, fille de carnavalier, engagée pour la cause du carnaval.

19. HAINARD Jacques \& KAEHR Roland (dir.), Collections passion, Neuchâtel, Musée d'ethnographie de Neuchâtel, 1982.

20. Le collectionneur n'a perçu qu'une seule subvention publique, plutôt modeste, du Département des Alpes-Maritimes.

21. Ceux-ci ont été l'objet d'une reconnaissance récente, par l'inscription à l'Inventaire national du patrimoine culturel immatériel des «Savoir-faire artisanaux associés au carnaval de Nice 
(Alpes-Maritimes)»(2018_67717_INV_PCI_FRANCE_00421) : https://www.culture.gouv.fr/Sitesthematiques/Patrimoine-culturel-immateriel/L-inventaire-national-du-PCI/Inventaire-national/ Pratiques-sociales-et-festives

22. DASSIÉ Véronique, Objets d'affection Une ethnologie de l'intime, Paris, Éditions du CTHS, coll. « Le regard de l'ethnologue ", 2010.

23. On remarque qu'il existait en fait deux sculptures de ce type et que la patte gauche du lion était alors positionnée différemment, par rapport à la fig. 5. Certainement a-t-elle été endommagée.

24. SAGNES Sylvie, «Au miroir de la médiation : le présent du patrimoine », in TORNATORE JeanLouis (dir.), Le Patrimoine comme expérience. Implications anthropologiques, Paris, Éditions de la Maison des sciences de l'homme, coll. "Ethnologie de la France et des mondes contemporains ", 2019, p. 69-90. Cette définition du patrimoine est directement issue des conceptions élaborées par Daniel Fabre.

\section{RÉSUMÉS}

Le carnaval de Nice est aujourd'hui l'un des carnavals les plus célèbres au monde. Au sein de cette festivité, les défilés de chars et de grosses têtes, accompagnés de troupes, s'imposent comme les événements phares. Chaque année, la thématique du carnaval change, ce qui implique de détruire les créations des défilés pour en refaire de nouvelles. Il s'agit donc d'œuvres éphémères. Cette configuration amène les fabricants à endosser le rôle de créateur-destructeur. L'éphémère est vécu en tant que réalité intrinsèque au carnaval, incarnant son cycle. Il peut aussi être loué comme une source de stimulation créative liée à la joie de créer et recréer, mais il peut, dans d'autres cas, générer de la tristesse et un sentiment d'injustice. Si la plupart des objets carnavalesques sont donc démontés et détruits, certains d'entre eux peuvent néanmoins connaître une autre destinée. Des sculptures de chars, des grosses têtes sont revendues, données, réutilisées à des fins décoratives et commerciales, ou encore exportées, mais également patrimonialisées, muséifiées. En reprenant le concept de biographie d'objets, l'auteur propose de mettre en lumière les trajectoires de ces objets festifs. Leurs réemplois et les usages qui en découlent reflètent les grandes dynamiques qui traversent ce carnaval urbain.

The Nice Carnival is currently one of the most famous carnivals in the world. During these festivities, the procession of carts and "big heads", along with companies, are key features of the event. Each year, the theme of the carnival changes, which implies the destruction of the works created for the previous processions, so as to build new ones. They are thus short-lived pieces of art. This leads the builders to be both creators and destroyers. The ephemeral aspect is experienced as an inherent reality of the carnival, an embodiment of its cyclical nature. It may also be praised as a source of creative stimulation, connected to the pleasure of creating and creating again, but it can be a source of sadness and feeling of injustice. While most of the objects of the carnival are dismantled and destroyed, some of them are likely to have another fate. The sculptures of carts or the "big heads" can be sold, given or reused with commercial or ornamental aims or even exported abroad, but also declared a part of heritage and sent to museums. By working on this concept of biographies of objects, the author offers to shed light on the future of these festive works. Their consequent reuse and the purposes of such mirror the large dynamics at work in this urban carnival. 
INDEX

Keywords : biography of objects, Nice Carnival, carnival members, circulation of objects, ephemeral, aesthetics, passion, heritage identification, Nice, reuse

Mots-clés : biographie d'objets, carnaval de Nice, carnavaliers, circulation d'objets, éphémère, esthétique, passion, patrimonialisation, Nice, réemplois

\section{AUTEUR}

\section{ANTONIN CHABERT}

Chercheur associé à l'Institut d'ethnologie méditerranéenne, européenne et comparative (Idemec), Aix-Marseille Université / CNRS, Aix-en-Provence.

antoninchabert@yahoo.fr 